 \\ HUDDERSFIELD
}

Mental Health and Learning Disabilities Research and Practice

Volume 3 Number 1 April 2006

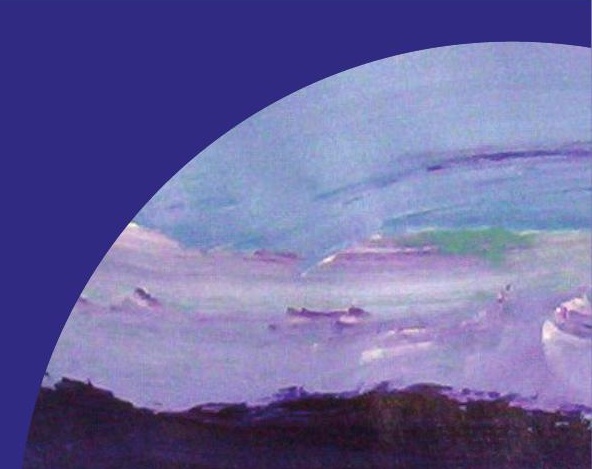




\title{
Intensive Interaction: a Research Review
}

\author{
Graham Firth $^{1}$
}

${ }^{1}$ Leeds Mental Health Teaching NHS Trust 


\title{
Intensive Interaction - a Research Review
}

\author{
Graham Firth
}

\begin{abstract}
Intensive Interaction is a socially interactive approach to supporting and developing the pre-verbal communication and sociability of people with severe or profound learning disabilities, or severe autism. Developed in the 1980's from the psychological model of 'augmented mothering' the approach currently has an increasing number of proponents who make claims for increased social responsiveness due to the use of the approach.

This short paper aims to evaluate some of the evidential claims of consequential increased social responses from people with severe and profound and multiple learning disabilities due to the use of Intensive Interaction techniques. Thus the paper presents a review of findings presented in relevant research papers which have been published in generally recognised academic journals.

From this review the author concludes that although expanding, the current body of research has been limited in scope and scale, and has generally been conducted by a small number of Intensive Interaction practitioners and advocates. However, increased client social responsiveness was consistently reported across the research projects reviewed.

In conclusion, this paper advocates for further systematic research into the approach by the wider research community to further develop the evidential base of the approach.
\end{abstract}

Key words: intensive interaction, learning disabilities, research review.

\section{Intensive Interaction: a Research Review}

According to the British Institute of Learning Disabilities (BILD) Intensive Interaction is:

'... a practical approach to interacting with people with learning disabilities who do not find it easy communicating or being social....' (accessed at www.bild.org.uk/factsheets/intensive interaction.htm on $\underline{16 / 02 / 06)}$.

Intensive Interaction was originally developed in the 1980's and was described by teachers Dave Hewett and Melanie Nind in 'Access to Communication: Developing the basics of communication with people with severe learning difficulties through Intensive Interaction' (1994). Eschewing the prevalent behaviourally inspired pedagogy and skill analysis model of curriculum formulation that prioritised self-help or independent living skills, Intensive Interaction instead aimed to facilitate the acquisition of fundamental communication skills and sociability of people with severe or profound and multiple learning difficulties (it has also been used with people with severe Autistic Spectrum Disorder). The approach was built on the concept of 'Augmented Mothering' developed by psychologist Gary 
Ephraim (1982), who argued for the use of naturalistic interactions with learning disabled clients based on 'infant-caregiver' interactional techniques.

The techniques of Intensive Interaction include the use of non-task associated physical proximity, responsive eye contact and facial signalling, physical contact, vocal echoing and behavioural mirroring. According to Hewett and Nind (1998),

'Intensive Interaction is characterised by regular, frequent interactions between the practitioner ... and individual with learning disabilities, in which there is no task or outcome focus, but in which the primary concern is the quality of the interaction itself' (p.2).

During the interactions the practitioner endeavours to follow the client's lead, thus responding contingently to their behaviour or activities to create a joint focus and increased social acknowledgement and interpersonal signalling (this generally being non-verbal in nature).

With increased use and advocacy across disciplines (e.g. teaching, speech \& language therapy, psychology, social care), Intensive Interaction is now seen more widely as a way of teaching fundamental communication, as a means of relationship building, or simply as a way of spending time with people who are remote or find difficulty in being social.

This article concerns itself with published research papers that are clearly focussed on the social responses of the learning disabled person to the approach. Appropriate papers for inclusion were identified by initially accessing a published list of specific Intensive Interaction research articles (via www.intensiveinteraction.co.uk ) combined with a process of actively following a reference trail through the appropriate papers. Specific papers were then selected to include papers from differing researchers or research teams, and to accommodate articles from a breadth of learning disability and special educational journals. Due to the brevity of this article only six papers are summarized below; three are studies that were carried out with adult participants and three with children.

\section{Research with Adults Participants}

The three papers briefly discussed below (Nind, 1996; Lovell, Jones and Ephraim, 1998; Elgie and Maguire, 2001) employ varying methodologies, and were carried out in different settings: an adult special educational arena; a long-stay residential hospital; and a small group home. However, across the studies there emerge the common themes of increased or novel interactive responses by the research participants with severe or profound and multiple learning disabilities. Collectively the papers' findings include increased frequency of social contact demonstrated through raised levels of contingent smiling, enhanced use of socially significant physical contact, raised levels of joint attention, and increased use of vocalisation.

In Nind's (1996) research ('Efficacy of Intensive Interaction: developing sociability and communication in people with severe and complex learning difficulties using an approach based on caregiver - infant interaction') six adult students with severe and complex learning difficulties, who attended a 
school within the grounds of a long stay residential hospital, were engaged with an Intensive Interaction intervention.

This research employed a 'multiple-baseline interrupted time series methodology'. Thus the students' interactional behaviour was recorded during both a 'base-line phase' (of up to six months) and an 'intervention phase' with daily sessions of Intensive Interaction (lasting up to 18 months). Data was generated by employing video analysis and a number of observation schedules, including the Pre-Verbal Communication Schedule (Kieran and Reid, 1987).

As a result of the Intensive Interaction, the researcher claimed that each student developed new interactive behaviour, such as 'contingent smiling', 'maintaining a state of joint focus' or 'contingent vocalisation'. More generally all of the students made advances in their communication abilities as measured on the Pre-Verbal Communication Schedule, with progress noted particularly in the areas of vocal imitation, communication through gesture, and communication through the use of sounds. According to the author, there were no significant events concurrent with the onset of the Intensive Interaction intervention, and therefore no rival explanations for the developments.

A study by Lovell, Jones, and Ephraim (1998) ('The Effect of Intensive Interaction on the Sociability of a Man with Severe Intellectual Disabilities') investigated the use of Intensive Interaction with a socially withdrawn, 53year-old man with severe intellectual disabilities who lived within a large residential hospital. The client was observed in two differing social scenarios: in one, a clinical psychologist engaged him with Intensive Interaction; in the second, the same psychologist remained close to the client but did not interact with him (these were termed 'proximity sessions'). Comparative observational data was gathered from each session using ' 10 second momentary time sampling' of video-recordings. The same method had previously been employed to gain base-line observations of the client in his normal environment, where he was usually observed to be sitting alone in a corner.

The findings of this research claimed to indicate that during the Intensive Interaction sessions the client initiated much more physical contact than prior to the intervention, when it was never evidenced. There were also claims for more physical contact during the Intensive Interaction than in the 'proximity sessions', where it was only occasionally observed. Also indicated was an increased time spent 'looking at people' during Intensive Interaction, and doing so with more consistency than in the 'proximity sessions'. No episodes of joint attention were recorded prior to the interventions and just one instance was recorded in the 'proximity sessions'. However, the client demonstrated many episodes of joint attention during the Intensive Interaction sessions.

The authors also claimed that there were additional benefits to the use of Intensive Interaction in terms of changes in the client's mood. The client was observed to smile, laugh and vocalise more in the Intensive Interaction sessions than prior to the interventions, when no examples of these responses were recorded. However, due to an unrelated injury to the client, this research endeavour had to be prematurely curtailed and so the findings should be judged as incomplete. 
A study by Elgie and Maguire (2001) ('Intensive Interaction with a Woman with Multiple and Profound Disabilities; a case study) reported on the use of Intensive Interaction with an extremely socially withdrawn woman with profound learning disabilities. This lady had engaged, since childhood, in serious self-injurious behaviour which damaged her face and eyes, and she was therefore blind. She had no verbal skills and was reported as using only very limited non-verbal communication.

After base-line measurements, a 16 week Intensive Interaction intervention was employed using physical contact (especially hand holding), vocal commentary with intonation, and sensitively timed vocal imitation.

According to the authors, one result of this intervention was increased spontaneous hand contact, which contrasted with the client's previous behaviour when no spontaneous reaching out had been observed. The authors also reported evidence of increased vocalisations during the Intensive Interaction sessions, as compared to when the lady was alone.

Somewhat disappointingly from the authors' perspective, there was no appreciable change observed in the level of the client's self injurious behaviour over time. However, the authors claimed that this was to be expected at such an early stage of the Intensive Interaction intervention.

\section{Research with Child Participants}

The claims made as a result of research into the responses of children to Intensive Interaction are similar to those for adults, with claims for increased social responsiveness. However, again it should be acknowledged that the body of research is not large.

The three papers summarised below were all carried out in an educational setting and employed varying methodologies. Again, common themes emerged indicating increased levels of joint attention, active participation and turn taking. However, one potentially important factor for research in this area is the consideration that children's development could be accounted for by a process of general maturation rather than any specific intervention. Therefore any causal links identified on the basis of evidence generated in this area should always be judged with this in mind.

One study into children's responses, carried out by Watson and Knight ((1991) 'An Evaluation of Intensive Interactive Teaching with Pupils with Very Severe Learning Difficulties') explored the use of Intensive Interaction with six pupils with severe learning difficulties at a special school in Scotland. The children's responses were studied over a whole school year. Six members of staff worked consistently with the same child, and used toys or objects to generate interactive games. Evidence was gathered using video recordings, contemporaneous staff records and interviews with staff members.

From this study the researchers claimed that staff-pupil pairs built up individual interaction styles that created potentially enabling changes in the children's social behaviour. Such developments for individual children included increased levels of alertness; increased instances of eye contact; increased turn taking and sharing of objects; increased socially significant 
vocalisations; increased active participation; and for one child, indications of improved gross motor control.

More subjectively, but perhaps equally as important, the staff emphasised that they believed that Intensive Interaction enabled the development of good pupil-staff relationships. The staff members also indicated other effects of Intensive Interaction as significant from their own perspective, including staff being more relaxed, tolerant and willing to wait for the children's responses.

With a view to generating more comparative data, Watson and Fisher ((1997) Evaluating the Effectiveness of Intensive Interaction Teaching with Pupils with Profound and Complex Learning Disabilities) evaluated two teaching methods, teacher-directed activity and Intensive Interaction, over a nine-month period, for pupils with very severe learning difficulties and multiple impairments. From Pre-verbal Communication Schedule assessments (Kiernan and Reid, 1987) and data from sessional videotapes, the authors claimed that there were some 'striking' examples of social or communicative behaviours evidenced during sessions of Intensive Interaction. It was also claimed that these behaviours were not observed during other, more didactic classroom activities. There was evidence of increased movement; the development of turn-taking sequences; and more pupil initiated activity.

Generally, according to the authors, during the Intensive Interaction sessions all the pupils 'demonstrated higher levels of active participation and enjoyment'. This contrasted with the teacher-directed group activities, where there was a tendency for pupils to take on the role of 'passive recipients' toward the activities. Based on these observations the authors concluded that Intensive Interaction was 'a more rewarding social experience' for the pupils, and one 'in which they showed initiative and control'.

A single case study by Kellett (2000) (Sam's Story: Evaluating Intensive Interaction in Terms of its Effect on the Social and Communicative ability of a Young Child with Severe Learning Difficulties) concerned a five-year-old boy, Sam, at a community special school (this paper reported on just one case out of a larger longitudinal study involving six children with severe learning disabilities). Sam often engaged in self-stimulatory behaviour and was indicated by the school staff as living 'in a world of his own'. He did not use any symbolic language or formal signs, made no eye contact with other people, and appeared not to observe nor respond to other peoples' facial signalling.

The researcher used a 'multiple-baseline interrupted time series' methodology, employing systematic video-recorded observation over one academic year. The observed progress made by Sam after the initiation of daily 10 minute sessions of Intensive Interaction was identified as:

- 'Modest progress' in the incidence of 'social physical contact'

- 'Clearly evident' progression for Sam in the incidence of 'eye contact'

- 'Huge steps' forward in 'looking at or towards a partner's face'

- Sam's ability or willingness to engage in joint focus or joint activity with the teacher 'developed dramatically' 
- Sam beginning 'to use his vocalising ability to respond contingently and to initiate contact'

It should be noted that the often emotive terminology used by the author in this paper stands in contrast to the more generally accepted scientific or quantitative vernacular. However, the author also wisely cautions against over-generalisation from such a single case study, identifying this paper as adding to the body of consistently reported increases in social responsiveness associated with the adoption of Intensive Interaction techniques.

\section{Discussion}

For pragmatic reasons such a brief paper as this has a limited scope, and therefore cannot claim to give a comprehensive view across all current research into Intensive Interaction. However, the body of evidence arising from the research papers reviewed in this article appears consistently supportive of claims of increased social responses from the research participants associated with the onset of Intensive Interaction interventions. There are repeated claims of novel or increased socially interactive behaviours manifest through greater use of eye contact; increased toleration of, or engagement in, physical contact activities; additional episodes of sustained joint attention; increased use of potentially communicative vocalisations; and an increased regard for facial signalling. There is also evidence of greater initiation of social interaction by some of the research participants.

However, in some aspects the findings from these research endeavours were not wholly consistent, and due to the different time scales employed not directly comparable. Nind (1996) makes a claim for reductions in the levels of the participants' ritualistic or self-stimulatory behaviours as new interactive behaviours emerge, whereas in the much shorter case study of Elgie and Maguire (2001) no significant change in the levels of their client's self-injurious behaviour was evidenced.

It could be argued that the body of evidence is still limited in its generalisabilty due to the methodologies employed, that is the use of smallscale or single case studies without experimental controls. The limited time scale of some of the research studies could also be seen as a potential weakness with respect to the validity of the findings in terms of sustainable changes to the behaviours measured. It should also be noted that the main body of research carried out into the use of Intensive Interaction has been conducted by a relatively small number of practitioners and advocates of the approach, generally educationalists and clinical psychologists. There is therefore also an issue of unintentional bias to be considered in the operationalisation and reporting of studies closely tied to the researchers own professional endeavours.

Another potential criticism of the body of research concerns the claims made concerning process. For those papers written from an explicitly educational perspective (and published in educational journals) there is an assumption, sometimes explicitly stated, that the novel or increased social responses arise out of a teaching or learning process, i.e. that there is specific social skill acquisition. The other, non-educational researchers 
seem to be indicating a much more rapid 'social inclusion' model of increased responsiveness, and indeed these research endeavours were generally much shorter, with social skill acquisition presumably much less likely within the given time frames (e.g. 10 or 16 weeks). Such a 'social inclusion' model seems to acknowledge the potential for the client or students' latent social skills or behaviour to be increasingly expressed as increased opportunities are presented to them in a socially inclusive way.

Some consideration within the research literature of cognitive or learning theory might help elucidate this apparent duality. Indeed some situated learning theories, e.g. Legitimate Peripheral Participation (Lave and Wenger, 1991) explicitly view authentic engagement in activities or 'joint enterprises' (in this case social and communicative enterprises) as a necessary precursor to conceptual development and skill acquisition. Thus, from such a perspective the educational or social processes underlying Intensive interaction could be viewed as seamless and phenomenologically co-dependant. Nevertheless, whatever the view taken on process, increased interactional activity has been systematically evidenced, but perhaps future research could benefit from being more conceptually aware of such a potential duality.

Taken together, despite the possible critiques and the potential for dual process, the research findings indicated in this review could be viewed as adding credible support to the case that if social occasions are purposefully generated in such a way as to facilitate the social participation of people with severe or profound learning disabilities, then this can lead to increased social engagement on their part.

Interestingly, despite Intensive Interaction being increasingly reported on, researched, and even indicated as accepted practice by a number of large voluntary and government agencies (British Institute of Learning Disabilities (ibid); The Foundation for People with Learning Disabilities (2001, p. 28); The Qualifications and Curriculum Authority (2001, p.18)) no large scale, centrally funded research into the approach has as yet been undertaken. But certainly it is this author's view that in times which increasingly focus on and value evidence-based practice, it is vital that further empirical research takes place to build on the already emergent body of evidence. However, such research would not only have to ensure robust validity and generalisability, but would also need to take complete account of the complexity of human interactional behaviour whilst acknowledging its full contextual depth.

Valuing People, the Government's Strategy for Learning Disability policy in the 21st Century, states that 'people with learning disabilities are often socially isolated' $(7: 39$, p. 81$)$. It also clearly states in Objective 7 that people should be enabled to '... develop a range of friendships, activities and relationships' (p. 127). If this objective is to genuinely transpire, particularly for those people with the most profound and complex learning disabilities, then further structured investigation into Intensive Interaction as a potential means for developing such activities, interests and relationships is undoubtedly required. 


\section{References}

British Institute of Learning Disabilities 2005 on-line at www.bild.org.uk: www.bild.org.uk/factsheets/intensive interaction.htm (accessed 2/07/05).

Department of Health 2001. Valuing People: A New Strategy for Learning Disability for the 21st Century [Cm 5086]. London: The Stationery Office.

Elgie, S. \& Maguire, N. 2001. Intensive Interaction with a woman with multiple and profound disabilities: a case study, Tizard Learning Disability Review, 6 (3), 18-24.

Ephraim, G. 1982. Developmental processes in mental handicap: a generative structure approach, Unpublished $\mathrm{PhD}$ thesis, Brunel University Foundation.

Hewett, D. \& Nind, M. 1998 (eds) Interaction in Action: reflections on the use of Intensive Interaction, David Fulton, London.

Kellett, M. 2000. Sam's story: evaluating Intensive Interaction in terms of its effect on the social and communicative ability of a young child with severe learning difficulties, Support for Learning, 15 (4), 165-171.

Kiernan, C. \& Reid, B. 1987. Pre-Verbal Communication Schedule. NFERNelson, Windsor.

Lave, J. \& Wenger, E. 1991. Situated Learning: Legitimate peripheral participation, University of Cambridge Press, Cambridge.

Lovell, D., Jones, R. \& Ephraim, G. 1998. The effect of intensive interaction on the sociability of a man with severe intellectual disabilities, The International Journal of Practical Approaches to Disability, 22 (2/3), 3-8.

Nind, M. \& Hewett, D. 1994. Access to Communication: Developing the basics of communication with people with severe learning difficulties through Intensive Interaction, David Fulton, London.

Nind, M. 1996. Efficacy of Intensive Interaction: developing sociability and communication in people with severe and complex learning difficulties using an approach based on caregiver- infant interaction, The European Journal of Special Educational Needs, 11 (1), 48-66.

The Qualifications and Curriculum Authority 2001. Planning, teaching and assessing the curriculum for pupils with learning difficulties: general guidelines, QCA Publications, London.

The Foundation for People with Learning Disabilities 2001. All About Autistic Spectrum Disorders, The Mental Health Foundation, London.

Watson J. \& Fisher A. 1997. Evaluating the effectiveness of Intensive Interaction teaching with pupils with profound and complex learning disabilities, The British Journal of Special Education, 24 (2), 80-87. 
Watson J. \& Knight C. 1991. An evaluation of Intensive Interactive teaching with pupils with very severe learning difficulties, Child Language Teaching and Therapy, 7 (3), 310-25. 